\title{
Intensive Care Unit Admission Patterns for Mild Traumatic Brain Injury in the USA
}

\author{
Robert H. Bonow ${ }^{1,2^{*}}$, Alex Quistberg ${ }^{1,3}$, Frederick P. Rivara ${ }^{1,4}$ and Monica S. Vavilala ${ }^{1,5}$
}

(c) 2018 Springer Science+Business Media, LLC, part of Springer Nature and Neurocritical Care Society

\begin{abstract}
Background: Patients with mild traumatic brain injury (TBI) are frequently admitted to an intensive care unit (ICU), but routine ICU use may be unnecessary. It is not clear to what extent this practice varies between hospitals.

Methods: We conducted a retrospective cohort study using the National Trauma Data Bank. Patients with at least one TBI ICD-9-CM diagnosis code, a head abbreviated injury score (AIS) $\leq 4$, and Glasgow coma scale (GCS) $\geq 13$ were included; individuals with only a concussion and those with a non-head AIS $>2$ were excluded. Primary outcomes were ICU admission and "overtriage" to the ICU, defined by: ICU stay $\leq 1$ day; hospital stay $\leq 2$ days; no intubation; no neurosurgery; and discharged to home. Mixed effects multivariable models were used to identify patient and facility characteristics associated with these outcomes.

Results: A total of 595,171 patients were included, $44.7 \%$ of whom were admitted to an ICU; $17.3 \%$ of these met the criteria for overtriage. Compared with adults, children $<2$ years were more likely to be admitted to an ICU (RR $1.21,95 \% \mathrm{Cl} 1.16-1.26$ ) and to be overtriaged (RR 2.06, 95\% Cl 1.88-2.25). Similarly, patients with isolated subarachnoid hemorrhage were at greater risk of both ICU admission (RR 2.36, 95\% Cl 2.31-2.41) and overtriage (RR 1.22, 95\% CI 1.17-1.28). The probabilities of ICU admission and overtriage varied as much as 16- and 11-fold across hospitals, respectively; median risk ratios were 1.67 and 1.53, respectively. The likelihood of these outcomes did not vary substantially with the characteristics of the treating facility.

Conclusions: There is considerable variability in ICU admission practices for mild TBI across the USA, and some of these patients may not require ICU-level care. Refined ICU use in mild TBI may allow for reduced resource utilization without jeopardizing patient outcomes.
\end{abstract}

Keywords: Traumatic brain injury, Intensive care units, Health resources

\section{Background}

Patients with traumatic brain injury (TBI) account for several hundred thousand hospital admissions annually in the USA [1]. The majority of these injuries are mild, traditionally defined by a Glasgow Coma Scale (GCS) score of 13 or greater [2]. While some patients with mild TBI (mTBI) require emergent neurosurgical intervention upon arrival to the hospital, most do not and are managed expectantly [3-7]. Occasionally, individuals

\footnotetext{
*Correspondence: rbonow@uw.edu

${ }^{1}$ Harborview Injury Prevention Research Center, Harborview Medical Center, Seattle, WA, USA

Full list of author information is available at the end of the article
}

with initially reassuring neurological examinations and imaging findings can deteriorate, requiring neurosurgical intervention, intubation, or other emergent lifesaving procedures [7]. For this reason, individuals with mTBI are often admitted to the intensive care unit (ICU) for close monitoring so that any deterioration can be quickly identified and acted upon.

The routine admission of patients with mTBI to the ICU constitutes a significant use of healthcare resources, and in some cases, the benefits are dubious [4-8]. Indeed, a meta-analysis of 49 studies found that the rates of clinical deterioration or death among patients with GCS $\geq 13$ were $11 \%$ and $1.4 \%$, respectively [8], suggesting that

\section{望 Springer}


the majority of these patients do not require such close observation. One large retrospective study conducted across eight level 1 trauma centers in the western USA found that only $5 \%$ of mild TBI patients with a GCS of 15 admitted to an ICU required ICU-level care, defined as neurosurgical intervention, vasopressor administration, invasive hemodynamic monitoring, mechanical ventilation, or blood product transfusion [7]. The same study also reported substantial variability in the triage patterns for patients with mTBI, with a 30 -fold difference in the adjusted odds of ICU admission between the facilities with the most aggressive and least aggressive ICU admission practices [7]. A separate analysis using prospectively collected TRACK-TBI pilot data similarly demonstrated that one of two participating centers was five times more likely to admit patients to the ICU [4]. Importantly, no study has demonstrated evidence of worse in-hospital or long-term outcomes when patients were triaged to the acute care ward rather than to the ICU. To the contrary, functional and neuropsychiatric outcomes were comparable [4].

Variable use of an intervention such as an ICU admission without evidence of differential outcomes indicates that there may be an opportunity to improve resource utilization by standardizing triage and admission practices. While it has been demonstrated that significant variability can exist between academic trauma centers within a geographic region [7], it is not known to what extent ICU admission practices vary on a national level and between facilities of different characteristics. Furthermore, the majority of studies published on this topic have not analyzed patients by the type of intracranial injury identified. This limitation is important because the risk of clinical deterioration and the probability of intervention vary considerably depending on the type of injury present [9-11].

This study was conducted to identify the clinical factors prompting admission to the ICU among individuals with mild complicated TBI, defined by a GCS of 13-15, a head Abbreviated Injury Scale (AIS) of less than five, and an abnormal computed tomography (CT) scan. We also sought to examine the extent to which these patients are "overtriaged" to the ICU, only to rapidly discharge to home without intervention. A final goal was to assess the variability of this practice around the country and to identify facility characteristics associated with an increased likelihood of ICU admission.

\section{Methods}

Data from the National Trauma Data Bank (NTDB) were used to conduct a retrospective cohort study spanning the years 2007 through 2014. Because the NTDB contains fully anonymized records, human subjects approval was not required for this study. Data regarding demographics, comorbidities, vital signs, GCS, injury mechanisms, injury diagnoses, inpatient procedures, hospital disposition, and facility characteristics were obtained from the database.

The NTDB records patient injuries using two diagnosis coding systems: the International Classification of Diseases, Ninth Revision, Clinical Modification (ICD-9-CM) and the AIS. While manually entered AIS codes contain granular details regarding injury location and severity that are lacking in ICD-9-CM diagnosis codes, the majority of records obtained from the NTDB contained only ICD-9-CM codes. To generate estimates of injury severity, we used the Stata package ICDPIC to map ICD-9-CM diagnosis codes to maximum AIS scores for each body region and to calculate an overall injury severity score (ISS). ICDPIC has been shown to have excellent discrimination for injury severity when applied to large datasets [12].

We included all patients with one or more of the standard ICD-9-CM diagnosis codes for TBI [13] (800.0-801.9, 803.0-804.9, 850.0-854.1, 950.1-950.3, 959.01, 995.55) who had a post-resuscitation emergency department (ED) GCS of 13 or greater and an AIS head of less than five. While recent research has increasingly categorized patients with a GCS of 13 as moderate TBI, this reclassification has not necessarily translated to clinical practice; GCS 13 and higher has long been used to define mild TBI and was thus the definition employed for the purposes of this study $[14,15]$.

Because significant extracranial injuries may influence the decision to admit a patient with mild TBI to the ICU, individuals with a maximum AIS greater than two in any other body region ( $\max$ non-head AIS) were excluded. We also excluded patients who died in the ED, required prehospital cardiopulmonary resuscitation, were intubated in the ED, were taken to surgery directly from the $E D$, or were discharged from the hospital without an inpatient admission following ED care. Because patients with concussion and no intracranial pathology are at very low risk of delayed deterioration and are frequently discharged to home from the ED [16], patients with no diagnoses other than concussion were also excluded. Two cohorts were constructed from the resulting sample based on their disposition from the ED: patients admitted to the acute care ward and patients admitted to the ICU. In general, the decision whether or not to admit individuals to the ICU or to the acute care ward is made after expert consultation and review of neuroimaging studies.

\section{Variable Definitions}

We also examined the extent to which patients admitted to the ICU may have been overtriaged (i.e., admitted to the ICU when acute care observation may have sufficed). A patient was considered to have been overtriaged if all 
the following conditions were met: admitted to the ICU from the ED; had an ICU length of stay less than or equal to 1 day; had a total hospital length of stay of 2 days or less; was not intubated at any point during the hospital stay; did not undergo neurosurgical intervention; and discharged from the hospital to home or self-care. For the purposes of classifying patients as overtriaged or not, the small number of records with missing values for length of stay and discharge disposition were assumed to have ICU length of stay $>1$ day, hospital length of stay $>2$ days, and a discharge disposition other than home; missing values for ventilator days were assumed to be zero. Neurosurgical intervention was defined as placement of an intracranial pressure monitor or surgery such as burr holes or craniotomy, which were identified using ICD-9-CM procedure codes $(01.09,01.10,01.16,01.17,01.26,02.21$, $01.23,01.24,01.25,02.02,02.12,02.13,01.32,01.39$, $01.53,01.31)$. Data regarding some critical care interventions, such as vasopressor use [17], were not available in the NTDB. This strict definition of an overtriaged admission was used in order to construct a group of patients who required little more than brief observation.

For the purposes of our analyses, hypotension was defined as a systolic blood pressure less than $90 \mathrm{mmHg}$ in patients 10 years and older; age-specific cutoffs based on the Pediatric Advanced Life Support guidelines were used for younger patients [18].

Because each patient may have multiple TBI diagnoses, there are a large number of possible permutations. To account for patients with multiple TBI diagnoses and to simplify our analysis, we created a variable with mutually exclusive categories for each diagnosis (for patients with a single TBI ICD-9-CM diagnosis code) and categories for multiple diagnoses (for patients with more than one TBI ICD-9-CM diagnosis code). This variable included seven categories for patients with a single TBI diagnosis code: skull fracture without intracranial hemorrhage (ICH); skull fracture with $\mathrm{ICH}$; subdural hematoma (SDH); subarachnoid hemorrhage (SAH); epidural hematoma (EDH); cerebral contusion; and other TBI (other and unspecified intracranial injury based on ICD-9-CM codes). Because of the large number of possible TBI diagnosis combinations in patients with multiple diagnoses, we sought to limit our analysis only to the permutations that were sufficiently frequent to merit analysis. To accomplish this, we included only combinations that constituted at least $2 \%$ of the sample; the only multiple diagnosis combination over the $2 \%$ cutoff was SDH with $\mathrm{SAH}$, which was added as an eighth category. All other rare combinations $(<2 \%$ or less) were combined into a ninth category called "multiple diagnoses."

\section{Statistical Analysis}

Demographics, clinical data, injury characteristics, and facility details were analyzed descriptively. To identify characteristics associated with a greater likelihood of ICU admission, a mixed-effect Poisson regression with robust standard errors was performed using a random intercept for each facility. Covariates included patient demographics (gender, age, race, insurance status), medical comorbidities (total number of comorbidities, the presence of a bleeding diathesis), clinical factors (the presence of hypotension, total GCS, suspected alcohol or drug use), injury details (TBI diagnosis, injury mechanism), facility characteristics (teaching status, region, number of total beds, number of ICU beds for trauma patients), and the year of admission. A second mixed-effect regression with a random intercept for each facility and the same covariate structure was performed to identify characteristics that were independently associated with overtriage to the ICU. Patients with missing values for covariates were excluded from these analyses. Once the multivariable models were created, the resulting intercepts for each facility were log-transformed to risk ratios and plotted from lowest to highest to visualize facility-to-facility variation. Finally, median risk ratios were calculated to demonstrate the extent to which inter-facility variation influenced the probability of ICU admission and overtriage. The median risk ratio is calculated by ranking facilities based on the risk of an outcome, calculating the risk differential of all pairwise comparisons between lowerand higher-ranked facilities, and then taking the median value of the resulting distribution. Conceptually, the median risk ratio corresponds to the median increase in risk of an outcome between a lower- and higher-ranked facility for a patient with a given set of covariates [19-21]. All data analysis was performed using Stata version 14 (StataCorp LLC, College Station, TX).

\section{Results}

We identified a total of $1,869,614$ patients with at least one ICD-9-CM diagnosis code for TBI in the NTDB dataset. A total of 948,436 of them had a mild isolated TBI, defined by a GCS of 13 or greater and a maximum AIS score of two or less in any body region other than the head. After excluding individuals who were taken directly to surgery from the ED and those with no TBI diagnosis other than concussion, the final cohort consisted of 595,171 patients.

Of the resulting cohort, $44.7 \%(n=265,857)$ were admitted to an ICU. Over $95 \%$ of these patients never underwent neurosurgical intervention and more than $90 \%$ never required mechanical ventilation (Table 1). A minority of patients had an ICU length of stay of 1 day or less and a total hospital length of stay of 2 days or less; 
Table 1 Proportion of ICU-admitted patients meeting the definition for overtriage

\begin{tabular}{lcl} 
& Number of patients & Percentage of all ICU admissions (\%) \\
\hline Total hospital length of stay $\leq 2$ days & 75,310 & 28.3 \\
ICU Length of stay $\leq 1$ day & 106,122 & 39.9 \\
Never intubated & 242,530 & 91.2 \\
No neurosurgical intervention & 254,557 & 95.7 \\
Discharged to home or self-care & 182,951 & 68.8 \\
Overtriaged (all of the above) & 45,953 & 17.3 \\
\hline
\end{tabular}

ICU intensive care unit

more than two-thirds of patients were discharged directly to home after their hospitalization. In total, $17.3 \%$ of patients admitted to the ICU $(n=45,953)$ met the criteria for overtriage.

The demographic, clinical, and facility characteristics of the overall sample are presented in Table 2. Patients admitted to the ICU tended to be older and to have more medical comorbidities than those admitted to the acute care ward. More ICU-admitted patients had a GCS $<15$ or an intracranial hemorrhage; SDH and EDH in particular were more common among those triaged to the ICU compared with those admitted to acute care.

Once admitted to the ICU, patients younger than 35 made up a greater proportion of those meeting our definition of overtriage than those not meeting these criteria (Table 3). Patients 55 and older made up a much smaller proportion of the overtriaged group than they did of the overall ICU admission cohort, due in large part to a greater proportion of these patients remaining in the hospital for a total of two or more days and/or discharging to a skilled nursing facility. For example, $66.7 \%$ of patients aged 85 and older who were admitted to the ICU ultimately required discharge to a facility compared with $17.5 \%$ of those $36-54$ years. Similarly, $85.0 \%$ of those 85 and older were hospitalized for longer than 2 days, whereas only $71.6 \%$ of $36-54$ years olds remained hospitalized for that duration. Patients with an intracranial hemorrhage or a GCS less than 15 were much less likely to have been overtriaged than those without hemorrhage and with a normal GCS (Table 3).

In multivariable analysis, age was a significant independent predictor of both ICU admission and overtriage to the ICU (Table 4). Pediatric patients aged $\leq 18$ years and those 55 and older were more likely to be admitted to the ICU. However, once admitted to the ICU, children $<2$ years were twice as likely to meet our criteria for overtriage compared with those aged 36-54 (RR 2.06, 95\% CI 1.88-2.25). Conversely, patients 55 and older were significantly less likely to have been overtriaged.

A number of other patient characteristics were independently associated with ICU admission and/or overtriage to the ICU (Table 4). For example, patients with multiple medical comorbidities, coagulopathies, hypotension, or depressed GCS were more likely to be admitted to the ICU than those without, and they were significantly less likely to have been overtriaged. This pattern was also present for most patients with any type of intracranial hemorrhage; a notable exception is patients with isolated $\mathrm{SAH}$, who had a higher risk of overtriage (RR 1.22, 95\% CI 1.17-1.28).

Facilities located outside of the Northeast were all more likely to admit patients to the ICU, and individuals receiving care in the Midwest and South were also more likely to have been overtriaged (Table 4). While there were some differences between facilities of varying sizes and trauma levels, no clear pattern was evident.

The predicted probability of ICU admission and overtriage for the average patient varied considerably among hospitals (Figs. 1a and 1b, respectively). The risk of ICU admission at the most aggressive facility (RR 2.74, 95\% CI 2.28-3.30) was 16-fold higher than at the most conservative facility (RR 0.17, 95\% CI 0.09-0.32); the median risk ratio for ICU admission between lower- and higherranked facilities was 1.67. Similarly, we observed an 11-fold difference between the hospitals with the highest risk of overtriage (RR 2.84, 95\% CI 2.32-2.84) and the lowest risk (RR 0.25 , 95\% CI 0.17-0.38); the median risk ratio for overtriage was 1.53 . There was not an obvious correlation between a facility's probabilities of ICU admission and overtriage (Fig. 1c).

\section{Discussion}

Using a national sample from the NTDB, we conducted a large retrospective study examining the patterns of ICU admission for mild TBI in the USA. We found that there was substantial variability between facilities with regard to patient triage from the emergency department. We also identified a number of patient characteristics that predicted a higher likelihood of ICU admission. Finally, we examined how commonly ICU admission may have been unnecessary by identifying patients with brief ICU 
Table 2 Clinical and demographic characteristics of mTBI patients admitted to acute care and to the ICU

\begin{tabular}{|c|c|c|c|}
\hline & \multicolumn{3}{|l|}{ ED disposition } \\
\hline & $\begin{array}{l}\text { Acute care admission } \\
(n=329,314)\end{array}$ & $\begin{array}{l}\text { ICU admission } \\
(n=265,857)\end{array}$ & $\begin{array}{l}\% \text { Difference } \\
(95 \% \mathrm{Cl})\end{array}$ \\
\hline \multicolumn{4}{|l|}{ Gender } \\
\hline Male & 202,087 (61.4\%) & $166,416(62.6 \%)$ & $1.2 \%(0.9 \%, 1.5 \%)$ \\
\hline \multicolumn{4}{|l|}{ Age } \\
\hline$<2$ years & $22,082(7.0 \%)$ & $11,375(4.5 \%)$ & $-2.4 \%(-2.5 \%, 2.3 \%)$ \\
\hline 2-10 years & $20,902(6.6 \%)$ & $12,290(4.8 \%)$ & $-1.7 \%(-1.8 \%,-1.6 \%)$ \\
\hline $11-18$ years & $22,641(7.2 \%)$ & $15,760(6.2 \%)$ & $-0.9 \%(-0.8 \%,-1.1 \%)$ \\
\hline 19-35 years & $61,550(19.5 \%)$ & $39,907(15.7 \%)$ & $-3.7 \%(-3.9 \%,-3.5 \%)$ \\
\hline $36-54$ years & $61,550(19.5 \%)$ & $46,094(18.1 \%)$ & $-1.4 \%(-1.5 \%,-1.2 \%)$ \\
\hline $55-69$ years & $47,999(15.2 \%)$ & $46,396(18.2 \%)$ & $2.9 \%(2.7 \%, 3.1 \%)$ \\
\hline 70-85 years & $56,614(17.9 \%)$ & $60,782(23.8 \%)$ & $5.7 \%(5.5 \%, 5.9 \%)$ \\
\hline $85+$ years & $22,524(7.1 \%)$ & $22,268(8.7 \%)$ & $1.5 \%(1.4 \%, 1.7 \%)$ \\
\hline \multicolumn{4}{|l|}{ Race/ethnicity } \\
\hline White & $228,239(72.0 \%)$ & $188,388(73.3 \%)$ & $1.6 \%(1.3 \%, 1.8 \%)$ \\
\hline African-American & $36,019(11.4 \%)$ & $24,586(9.6 \%)$ & $-1.7 \%(-1.8 \%,-1.5 \%)$ \\
\hline Hispanic & $34,200(10.8 \%)$ & $28,342(11.0 \%)$ & $0.2 \%(0.1 \%, 0.4 \%)$ \\
\hline Asian & $6392(2.0 \%)$ & $5948(2.3 \%)$ & $0.3 \%(0.2 \%, 0.4 \%)$ \\
\hline Other & $12,107(3.8 \%)$ & $9796(3.8 \%)$ & $0.0 \%(0.0 \%, 0.1 \%)$ \\
\hline \multicolumn{4}{|l|}{ Payer } \\
\hline Private & $83,362(28.3 \%)$ & $63,622(26.0 \%)$ & $-1.4 \%(-1.6 \%,-1.2 \%)$ \\
\hline Medicare & $96,913(32.9 \%)$ & $96,608(39.5 \%)$ & $6.9 \%(6.7 \%, 7.1 \%)$ \\
\hline Medicaid & $42,759(14.5 \%)$ & $29,944(12.2 \%)$ & $-1.7 \%(-1.9 \%,-1.6 \%)$ \\
\hline Self-pay/not billed & $41,229(14.0 \%)$ & $31,215(12.7 \%)$ & $-1.3 \%(-1.4 \%,-1.1 \%)$ \\
\hline Auto & $13,588(4.6 \%)$ & $9291(3.8 \%)$ & $-0.8 \%(-1.0 \%,-0.6 \%)$ \\
\hline Worker comp & $5649(1.9 \%)$ & $4300(1.8 \%)$ & $-0.1 \%(-0.2 \%, 0.0 \%)$ \\
\hline Other & $11,347(3.8 \%)$ & $9874(4.0 \%)$ & $0.2 \%(0.1 \%, 0.3 \%)$ \\
\hline \multicolumn{4}{|l|}{ Number of comorbidities } \\
\hline 0 & $170,864(51.9 \%)$ & $112,665(42.4 \%)$ & $-9.5 \%(-9.8 \%,-9.3 \%)$ \\
\hline 1 & 75,395 (22.9\%) & $64,954(24.4 \%)$ & $1.5 \%(1.3 \%, 1.8 \%)$ \\
\hline$\geq 2$ & $83,055(25.2 \%)$ & $88,238(33.2 \%)$ & $8.0 \%(7.7 \%, 8.2 \%)$ \\
\hline \multicolumn{4}{|l|}{ Transferred in } \\
\hline Yes & $118,400(36.0 \%)$ & $101,511(38.2 \%)$ & $2.2 \%(2.0 \%, 2.5 \%)$ \\
\hline \multicolumn{4}{|l|}{ Hypotensive } \\
\hline Yes & $2415(0.8 \%)$ & $2439(0.9 \%)$ & $0.1 \%(0.1 \%, 0.2 \%)$ \\
\hline \multicolumn{4}{|l|}{ Mechanisms } \\
\hline Fall & $175,519(53.3 \%)$ & $156,783(59.0 \%)$ & $5.7 \%(5.4 \%, 5.9 \%)$ \\
\hline Motorcycle & $9779(3.0 \%)$ & $7911(3.0 \%)$ & $0.0 \%(0.0 \%, 0.0 \%)$ \\
\hline Motor vehicle collision & $47,993(14.6 \%)$ & $31,307(11.8 \%)$ & $-2.8 \%(-3.0 \%,-2.6 \%)$ \\
\hline Pedestrian & $22,583(6.9 \%)$ & $17,725(6.7 \%)$ & $-0.2 \%(-0.3 \%, 0.0 \%)$ \\
\hline Struck by & $41,501(12.6 \%)$ & $27,242(10.2 \%)$ & $-2.4 \%(-2.5 \%,-2.2 \%)$ \\
\hline Other & $39,288(11.9 \%)$ & $29,743(11.2 \%)$ & $-0.7 \%(-0.9 \%,-0.5 \%)$ \\
\hline \multicolumn{4}{|l|}{ Injury type } \\
\hline Blunt & $311,691(99.1 \%)$ & $251,469(98.8 \%)$ & $-0.3 \%(-0.4 \%,-0.2 \%)$ \\
\hline Penetrating & $2819(0.9 \%)$ & $3114(1.2 \%)$ & $0.3 \%(0.3 \%, 0.4 \%)$ \\
\hline \multicolumn{4}{|l|}{ Injury intent } \\
\hline Unintentional & $286,587(87.9 \%)$ & $235,309(89.3 \%)$ & $1.4 \%(1.3 \%, 1.6 \%)$ \\
\hline Assault & $38,760(11.9 \%)$ & $27,148(10.3 \%)$ & $-1.6 \%(-1.7 \%,-1.4 \%)$ \\
\hline Self-inflicted & $770(0.2 \%)$ & $1044(0.4 \%)$ & $0.2 \%(0.1 \%, 0.2 \%)$ \\
\hline
\end{tabular}


Table 2 continued

\begin{tabular}{|c|c|c|c|}
\hline & \multicolumn{3}{|l|}{ ED disposition } \\
\hline & $\begin{array}{l}\text { Acute care admission } \\
(n=329,314)\end{array}$ & $\begin{array}{l}\text { ICU admission } \\
(n=265,857)\end{array}$ & $\begin{array}{l}\% \text { Difference } \\
(95 \% \mathrm{Cl})\end{array}$ \\
\hline \multicolumn{4}{|l|}{ Max non-head AIS } \\
\hline 0 & $103,473(31.4 \%)$ & $88,883(33.4 \%)$ & $2.0 \%(1.7 \%, 2.3 \%)$ \\
\hline 1 & $126,490(38.4 \%)$ & $103,178(38.8 \%)$ & $0.4 \%(0.2 \%, 0.6 \%)$ \\
\hline 2 & $99,350(30.2 \%)$ & $73,792(27.8 \%)$ & $-2.4 \%(-2.6 \%,-2.2 \%)$ \\
\hline \multicolumn{4}{|l|}{ ED composite GCS } \\
\hline 15 & $279,989(85.0 \%)$ & $197,792(74.4 \%)$ & $-10.6 \%(-10.8 \%,-10.4 \%)$ \\
\hline 14 & $41,439(12.6 \%)$ & $53,323(20.1 \%)$ & $7.5 \%(7.3 \%, 7.7 \%)$ \\
\hline 13 & 7886 (2.4\%) & $14,742(5.5 \%)$ & $3.1 \%(3.0 \%, 3.3 \%)$ \\
\hline \multicolumn{4}{|l|}{ Injury diagnosis } \\
\hline Skull fracture without ICH & $65,825(20.0 \%)$ & $16,514(6.2 \%)$ & $-13.8 \%(-13.9 \%,-13.6 \%)$ \\
\hline Skull fracture with ICH & $27,516(8.4 \%)$ & $34,251(12.9 \%)$ & $4.5 \%(4.4 \%, 4.7 \%)$ \\
\hline $\mathrm{EDH}$ & $2341(0.7 \%)$ & $3556(1.3 \%)$ & $0.6 \%(0.6 \%, 0.7 \%)$ \\
\hline $\mathrm{SDH}$ & $50,393(15.3 \%)$ & $59,420(22.4 \%)$ & $7.1 \%(6.9 \%, 7.2 \%)$ \\
\hline $\mathrm{SAH}$ & $37,635(11.4 \%)$ & $35,010(13.2 \%)$ & $1.7 \%(1.6 \%, 1.9 \%)$ \\
\hline SAH and SDH & 9401 (2.9\%) & $16,000(6.0 \%)$ & $3.2 \%(3.1 \%, 3.3 \%)$ \\
\hline Cerebral contusion & $16,696(5.1 \%)$ & $13,815(5.2 \%)$ & $0.1 \%(0.0 \%, 0.2 \%)$ \\
\hline Other TBI & $88,142(26.8 \%)$ & $26,523(10.0 \%)$ & $-16.8 \%(-17.0 \%,-16.6 \%)$ \\
\hline Multiple diagnoses & $31,365(9.5 \%)$ & $60,768(22.9 \%)$ & $133 \%(13.1 \%, 13.5 \%)$ \\
\hline \multicolumn{4}{|l|}{ Hospital teaching status } \\
\hline Community & $108,833(33.0 \%)$ & 106,015 (39.9\%) & $6.9 \%(6.6 \%, 7.1 \%)$ \\
\hline Non-teaching & $35,576(10.8 \%)$ & $34,489(13.0 \%)$ & $2.2 \%(2.0 \%, 2.3 \%)$ \\
\hline University & $184,905(56.1 \%)$ & $125,353(47.2 \%)$ & $-8.9 \%(-9.3 \%,-8.7 \%)$ \\
\hline \multicolumn{4}{|l|}{ Hospital region } \\
\hline Northeast & $69,531(21.6 \%)$ & $46,618(17.8 \%)$ & $-3.8 \%(-3.9 \%,-3.5 \%)$ \\
\hline Midwest & $87,879(27.3 \%)$ & $67,214(25.7 \%)$ & $-1.6 \%(-1.8 \%,-1.4 \%)$ \\
\hline West & $61,964(19.2 \%)$ & $62,565(23.9 \%)$ & $4.7 \%(4.5 \%, 4.9 \%)$ \\
\hline South & $103,042(32.0 \%)$ & $85,563(32.7 \%)$ & $0.7 \%(0.6 \%, 0.9 \%)$ \\
\hline \multicolumn{4}{|l|}{ Hospital size } \\
\hline$\leq 200$ beds & $15,874(4.8 \%)$ & $11,865(4.5 \%)$ & $-0.3 \%(-0.4 \%,-0.3 \%)$ \\
\hline $201-400$ beds & $86,670(26.3 \%)$ & $72,808(27.4 \%)$ & $1.1 \%(0.8 \%, 1.3 \%)$ \\
\hline $401-600$ beds & $100,223(30.4 \%)$ & $85,110(32.0 \%)$ & $1.6 \%(1.3 \%, 1.8 \%)$ \\
\hline$>600$ beds & $126,547(38.4 \%)$ & $96,074(36.1 \%)$ & $-2.3 \%(-2.5 \%, 2.0 \%)$ \\
\hline \multicolumn{4}{|l|}{ Number of trauma ICU beds } \\
\hline$\leq 10$ beds & $25,741(7.8 \%)$ & $18,289(6.9 \%)$ & $-0.9 \%(-1.1 \%,-0.8 \%)$ \\
\hline $11-25$ beds & $135,815(41.3 \%)$ & $107,854(40.6 \%)$ & $-0.7 \%(-0.9 \%,-0.4 \%)$ \\
\hline$>26$ beds & $167,522(50.9 \%)$ & $139,459(52.5 \%)$ & $1.6 \%(1.3 \%, 1.8 \%)$ \\
\hline
\end{tabular}

AIS abbreviated injury scale, ED emergency department, EDH epidural hematoma, GCS Glasgow coma scale, ICH intracranial hemorrhage, ICU intensive care unit, IPH intraparenchymal hemorrhage, $m T B I$ mild traumatic brain injury, $M V C$ motor vehicle collision, $S A H$ subarachnoid hemorrhage, $S D H$ subdural hematoma, $T B I$ traumatic brain injury

and hospital stays who did not require neurosurgical intervention and who discharged to self-care.

After adjusting for covariates, pediatric patients were significantly more likely to be admitted to the ICU than adults $36-54$ years, but they were also twice as likely as adults 36-54 years old to meet our definition of overtriage. This finding suggests that a substantial portion of pediatric ICU admissions for mild TBI are unnecessary. Heightened vigilance among practitioners caring for children may be responsible. Indeed, the majority of pediatric trauma care is delivered at hospitals without a pediatric trauma designation [22], and providers who do not typically care for young children may err on the side of caution by triaging these patients to the ICU. These 
Table 3 Clinical and demographic characteristics of ICU-admitted mTBI patients who did and did not meet the criteria for overtriage

\begin{tabular}{|c|c|c|c|}
\hline & \multicolumn{3}{|c|}{ Overtriage among those admitted to ICU } \\
\hline & Not overtriaged & Overtriaged & \% Difference \\
\hline & $(n=219,904)$ & $(n=45,953)$ & $(95 \% \mathrm{Cl})$ \\
\hline \multicolumn{4}{|l|}{ Gender } \\
\hline Male & $135,643(61.7 \%)$ & $30,773(67.0 \%)$ & $5.3 \%(4.8 \%, 5.8 \%)$ \\
\hline \multicolumn{4}{|l|}{ Age } \\
\hline$<2$ years & $6487(3.1 \%)$ & $4888(10.8 \%)$ & $7.7 \%(7.4 \%, 8.0 \%)$ \\
\hline $2-10$ years & $7168(3.4 \%)$ & $5122(11.3 \%)$ & $7.9 \%(7.6 \%, 8.2 \%)$ \\
\hline $11-18$ years & $10,846(5.2 \%)$ & $4914(10.8 \%)$ & $5.6 \%(5.4 \%, 6.0 \%)$ \\
\hline 19-35 years & $30,691(14.6 \%)$ & $9216(20.3 \%)$ & $6.3 \%(5.7 \%, 6.5 \%)$ \\
\hline $36-54$ years & $38,050(18.2 \%)$ & $8044(17.7 \%)$ & $-0.5 \%(0.7 \%,-0.3 \%)$ \\
\hline $55-69$ years & $39,985(19.1 \%)$ & $6411(14.1 \%)$ & $-5.0 \%(-5.3 \%,-4.7 \%)$ \\
\hline $70-85$ years & $55,388(26.4 \%)$ & $5394(11.9 \%)$ & $-14.5 \%(-14.8 \%,-14.2 \%)$ \\
\hline $85+$ years & $20,923(10.0 \%)$ & $1345(3.0 \%)$ & $-7.0 \%(-7.2 \%,-6.8 \%)$ \\
\hline \multicolumn{4}{|l|}{ Race/ethnicity } \\
\hline White & $157,778(74.1 \%)$ & $30,610(69.2 \%)$ & $-4.9 \%(-5.5 \%,-4.5 \%)$ \\
\hline African-American & $20,527(9.6 \%)$ & 4059 (9.2\%) & $-0.4 \%(-0.7 \%,-0.2 \%)$ \\
\hline Hispanic & $22,089(10.4 \%)$ & $6253(14.1 \%)$ & $3.7 \%(3.2 \%, 3.9 \%)$ \\
\hline Asian & 4815 (2.3\%) & $1133(2.6 \%)$ & $0.3 \%(0.1 \%, 0.4 \%)$ \\
\hline Other & $7597(3.6 \%)$ & $2199(5.0 \%)$ & $1.4 \%(1.1 \%, 1.5 \%)$ \\
\hline \multicolumn{4}{|l|}{ Payer } \\
\hline Private & $48,586(24.0 \%)$ & $15,036(35.6 \%)$ & $11.6 \%(11.2 \%, 12.1 \%)$ \\
\hline Medicare & $87,642(43.2 \%)$ & 8966 (21.2\%) & $-22.0 \%(-22.4 \%,-21.6 \%)$ \\
\hline Medicaid & $22,738(11.2 \%)$ & $7206(17.1 \%)$ & $4.9 \%(4.6 \%, 5.3 \%)$ \\
\hline Self-pay/not billed & $24,569(12.1 \%)$ & $6646(15.7 \%)$ & $3.6 \%(3.3 \%, 4.0 \%)$ \\
\hline Auto & 7725 (3.8\%) & $1566(3.7 \%)$ & $-0.1 \%(-0.3 \%, 0.0 \%)$ \\
\hline Worker comp & $3630(1.8 \%)$ & $670(1.6 \%)$ & $-0.2 \%(-0.3 \%,-0.1 \%)$ \\
\hline Other & $7763(3.8 \%)$ & $2111(5.0 \%)$ & $1.2 \%(1.0 \%, 1.4 \%)$ \\
\hline \multicolumn{4}{|l|}{ Number of comorbidities } \\
\hline 0 & 84,538 (38.4\%) & $28,127(61.2 \%)$ & $22.8 \%(22.3 \%, 23.3 \%)$ \\
\hline 1 & $54,904(25.0 \%)$ & $10,050(21.9 \%)$ & $-3.1 \%(-3.5 \%,-2.7 \%)$ \\
\hline$\geq 2$ & $80,462(36.6 \%)$ & 7776 (16.9\%) & $-19.7 \%(-20.1 \%,-19.3 \%)$ \\
\hline \multicolumn{4}{|l|}{ Transferred in } \\
\hline Yes & $81,766(37.2 \%)$ & $19,745(43.0 \%)$ & $5.8 \%(5.3 \%, 6.3 \%)$ \\
\hline \multicolumn{4}{|l|}{ Hypotensive } \\
\hline Yes & $2103(1.0 \%)$ & $336(0.8 \%)$ & $-0.2 \%(-0.3 \%,-0.1 \%)$ \\
\hline \multicolumn{4}{|l|}{ Mechanisms } \\
\hline Fall & $133,114(60.5 \%)$ & $23,669(51.5 \%)$ & $-9.0 \%(-9.5 \%,-8.5 \%)$ \\
\hline Motorcycle & $6626(3.0 \%)$ & $1285(2.8 \%)$ & $-0.2 \%(-0.3 \%, 0.0 \%)$ \\
\hline Motor vehicle collision & $25,437(11.6 \%)$ & $5870(12.8 \%)$ & $1.2 \%(0.9 \%, 1.5 \%)$ \\
\hline Pedestrian & $14,112(6.4 \%)$ & $3613(7.9 \%)$ & $1.5 \%(1.2 \%, 1.7 \%)$ \\
\hline Struck by & 20,367 (9.3\%) & $6875(15.0 \%)$ & $5.7 \%(5.4 \%, 6.0 \%)$ \\
\hline Other & $23,890(10.9 \%)$ & $5853(12.7 \%)$ & $1.8 \%(1.5 \%, 2.2 \%)$ \\
\hline \multicolumn{4}{|l|}{ Injury type } \\
\hline Blunt & $207,739(98.8 \%)$ & 43,730 (98.9\%) & $0.1 \%(0.0 \%, 0.1 \%)$ \\
\hline Penetrating & $2622(1.2 \%)$ & $492(1.1 \%)$ & $-0.1 \%(-0.2 \%, 0.0 \%)$ \\
\hline \multicolumn{4}{|l|}{ Injury intent } \\
\hline Unintentional & $195,341(89.7 \%)$ & 39,968 (87.6\%) & $-2.1 \%(-2.4,-1.8 \%)$ \\
\hline Assault & $21,563(9.9 \%)$ & 5585 (12.2\%) & $2.3 \%(2.0 \%, 2.7 \%)$ \\
\hline
\end{tabular}


Table 3 continued

\begin{tabular}{|c|c|c|c|}
\hline & \multicolumn{3}{|c|}{ Overtriage among those admitted to ICU } \\
\hline & $\begin{array}{l}\text { Not overtriaged } \\
(n=219,904)\end{array}$ & $\begin{array}{l}\text { Overtriaged } \\
(n=45,953)\end{array}$ & $\begin{array}{l}\% \text { Difference } \\
(95 \% \mathrm{CI})\end{array}$ \\
\hline Self-inflicted & $961(0.4 \%)$ & $83(0.2 \%)$ & $-0.2 \%(-0.3 \%,-0.2 \%)$ \\
\hline \multicolumn{4}{|l|}{ Max non-head AIS } \\
\hline 0 & $71,922(32.7 \%)$ & $16,961(36.9 \%)$ & $4.2 \%(3.7 \%, 4.7 \%)$ \\
\hline 1 & $81,906(37.2 \%)$ & $21,272(46.3 \%)$ & $9.1 \%(8.7 \%, 9.5 \%)$ \\
\hline 2 & $66,072(30.0 \%)$ & $7720(16.8 \%)$ & $-13.2 \%(-13.6 \%, 12.9 \%)$ \\
\hline \multicolumn{4}{|l|}{ ED composite GCS } \\
\hline 15 & $158,415(72.0 \%)$ & $39,377(85.7 \%)$ & $13.7 \%(13.2 \%, 14.0 \%)$ \\
\hline 14 & $47,835(21.8 \%)$ & $5488(11.9 \%)$ & $-9.9 \%(-10.2 \%,-9.5 \%)$ \\
\hline 13 & $13,654(6.2 \%)$ & $1088(2.4 \%)$ & $-3.8 \%(-4.0,-3.7 \%)$ \\
\hline \multicolumn{4}{|l|}{ Injury diagnosis } \\
\hline Skull fracture without ICH & $11,240(5.1 \%)$ & $5274(11.5 \%)$ & $6.4 \%(6.1 \%, 6.7 \%)$ \\
\hline Skull fracture with $\mathrm{ICH}$ & $27,887(12.7 \%)$ & $6364(13.8 \%)$ & $1.1 \%(0.8 \%, 1.5 \%)$ \\
\hline $\mathrm{EDH}$ & $2883(1.3 \%)$ & $673(1.5 \%)$ & $0.2 \%(0.0 \%, 0.3 \%)$ \\
\hline $\mathrm{SDH}$ & $51,145(23.3 \%)$ & $8275(18.0 \%)$ & $-5.3 \%(-5.6 \%,-4.9 \%)$ \\
\hline $\mathrm{SAH}$ & $26,993(12.3 \%)$ & $8017(17.4 \%)$ & $5.1 \%(4.8 \%, 5.5 \%)$ \\
\hline SAH and SDH & $14,290(6.5 \%)$ & $1710(3.7 \%)$ & $-2.8 \%(-2.9 \%,-2.6 \%)$ \\
\hline Cerebral contusion & $10,624(4.8 \%)$ & $3191(6.9 \%)$ & $2.1 \%(1.9 \%, 2.4 \%)$ \\
\hline Other TBI & $20,723(9.4 \%)$ & $5800(12.6 \%)$ & $3.2 \%(2.9 \%, 3.5 \%)$ \\
\hline Multiple diagnoses & $54,119(24.6 \%)$ & $6649(14.5 \%)$ & $-10.1 \%(-10.5,-9.8 \%)$ \\
\hline \multicolumn{4}{|l|}{ Hospital teaching status } \\
\hline Community & $88,480(40.2 \%)$ & 17,535 (38.2\%) & $-2.1 \%(-2.6 \%,-1.6 \%)$ \\
\hline Non-teaching & $29,165(13.3 \%)$ & $5324(11.6 \%)$ & $-1.7 \%(-2.0 \%,-1.4 \%)$ \\
\hline University & $102,259(46.5 \%)$ & $23,094(50.3 \%)$ & $3.8 \%(3.3 \%, 4.3 \%)$ \\
\hline \multicolumn{4}{|l|}{ Hospital region } \\
\hline Northeast & $39,010(18.0 \%)$ & 7608 (16.8\%) & $-1.2 \%(-1.6 \%,-0.8 \%)$ \\
\hline Midwest & $55,979(25.8 \%)$ & 11,235 (24.8\%) & $-1.0 \%(-1.4 \%,-0.6 \%)$ \\
\hline West & $49,345(22.8 \%)$ & $13,220(29.2 \%)$ & $6.4 \%(5.9 \%, 6.8 \%)$ \\
\hline South & $72,310(33.4 \%)$ & $13,253(29.2 \%)$ & $-4.2 \%(-4.6 \%,-3.7 \%)$ \\
\hline \multicolumn{4}{|l|}{ Hospital size } \\
\hline$\leq 200$ beds & $9292(4.2 \%)$ & $2573(5.6 \%)$ & $1.4 \%(1.1 \%, 1.6 \%)$ \\
\hline $201-400$ beds & $60,042(27.3 \%)$ & $12,766(27.8 \%)$ & $0.5 \%(0.0 \%, 0.9 \%)$ \\
\hline $401-600$ beds & $69,528(31.6 \%)$ & $15,582(33.9 \%)$ & $2.3 \%(1.8 \%, 2.8 \%)$ \\
\hline$>600$ beds & $81,042(36.9 \%)$ & $15,032(32.7 \%)$ & $-4.2 \%(-4.6 \%,-3.7 \%)$ \\
\hline \multicolumn{4}{|l|}{ Number of trauma ICU beds } \\
\hline$\leq 10$ beds & 15,070 (6.9\%) & $3219(7.0 \%)$ & $0.1 \%(-0.1 \%, 0.4 \%)$ \\
\hline $11-25$ beds & $88,843(40.4 \%)$ & $19,011(41.4 \%)$ & $1.0 \%(0.5 \%, 1.5 \%)$ \\
\hline$>26$ beds & $115,803(52.7 \%)$ & $23,656(51.6 \%)$ & $-1.1 \%(-1.7 \%, 0.7 \%)$ \\
\hline
\end{tabular}

AIS abbreviated injury scale, ED emergency department, EDH epidural hematoma, GCS Glasgow coma scale, ICH intracranial hemorrhage, ICU intensive care unit, IPH intraparenchymal hemorrhage, $m T B I$ mild traumatic brain injury, $M V C$ motor vehicle collision, $S A H$ subarachnoid hemorrhage, $S D H$ subdural hematoma, $T B I$ traumatic brain injury

tendencies could be amplified by the difficulty in obtaining a reassuring neurological examination in children too young to follow commands. Given concerns regarding the effects of ionizing radiation in children, close observation in an ICU may also be used in lieu of repeat CT scans.
By contrast, patients 55 and older were slightly more likely to be admitted to the ICU, but substantially less likely to be overtriaged than adults 36-54 years old. This difference is likely in part due to the greater need for post-discharge facility placement and the concomitant increase in length of stay (thus not meeting our 
Table 4 Multivariable models for ICU admission and overtriage to the ICU

\begin{tabular}{|c|c|c|c|c|c|c|c|c|}
\hline \multirow{3}{*}{ Age } & \multicolumn{4}{|c|}{ Admission to the ICU from the ED } & \multicolumn{4}{|c|}{ Overtriage among those admitted to ICU } \\
\hline & \multirow[t]{2}{*}{ RR } & \multicolumn{2}{|l|}{$95 \% \mathrm{Cl}$} & \multirow[t]{2}{*}{$P$} & \multirow[t]{2}{*}{$\mathbf{R R}$} & \multicolumn{2}{|l|}{$95 \% \mathrm{Cl}$} & \multirow[t]{2}{*}{$P$} \\
\hline & & & & & & & & \\
\hline$<2$ years & 1.21 & 1.16 & 1.26 & $<0.001$ & 2.06 & 1.88 & 2.25 & $<0.001$ \\
\hline $2-10$ years & 1.28 & 1.23 & 1.32 & $<0.001$ & 1.85 & 1.70 & 2.02 & $<0.001$ \\
\hline $11-18$ years & 1.12 & 1.09 & 1.15 & $<0.001$ & 1.45 & 1.35 & 1.55 & $<0.001$ \\
\hline 19-35 years & 0.96 & 0.95 & 0.98 & $<0.001$ & 1.23 & 1.19 & 1.27 & $<0.001$ \\
\hline $36-54$ years & [ref] & & & & [ref] & & & \\
\hline $55-69$ years & 1.06 & 1.04 & 1.08 & $<0.001$ & 0.86 & 0.83 & 0.89 & $<0.001$ \\
\hline $70-85$ years & 1.08 & 1.07 & 1.10 & $<0.001$ & 0.61 & 0.58 & 0.64 & $<0.001$ \\
\hline $85+$ years & 1.05 & 1.03 & 1.08 & $<0.001$ & 0.42 & 0.39 & 0.45 & $<0.001$ \\
\hline \multicolumn{9}{|l|}{ Comorbidities } \\
\hline 0 & [ref] & & & & [ref] & & & \\
\hline 1 & 1.03 & 1.02 & 1.04 & $<0.001$ & 0.91 & 0.89 & 0.94 & $<0.001$ \\
\hline$\geq 2$ & 1.06 & 1.04 & 1.07 & $<0.001$ & 0.67 & 0.64 & 0.70 & $<0.001$ \\
\hline Coagulopathy & 1.18 & 1.16 & 1.20 & $<0.001$ & 0.74 & 0.67 & 0.80 & $<0.001$ \\
\hline \multicolumn{9}{|l|}{ Injury intent } \\
\hline Unintentional & [ref] & & & & [ref] & & & \\
\hline Assault & 0.92 & 0.90 & 0.94 & $<0.001$ & 1.12 & 1.08 & 1.16 & $<0.001$ \\
\hline Self-inflicted & 1.15 & 1.06 & 1.26 & 0.001 & 0.32 & 0.22 & 0.45 & $<0.001$ \\
\hline Transferred in & 1.04 & 1.03 & 1.05 & $<0.001$ & 1.17 & 1.14 & 1.20 & $<0.001$ \\
\hline Alcohol or drug use & 1.08 & 1.06 & 1.09 & $<0.001$ & 1.10 & 1.07 & 1.14 & $<0.001$ \\
\hline Hypotension in the ED & 1.11 & 1.06 & 1.16 & $<0.001$ & 0.85 & 0.77 & 0.94 & 0.001 \\
\hline \multicolumn{9}{|l|}{ Max non-head AIS } \\
\hline 0 & [ref] & & & & [ref] & & & \\
\hline 1 & 0.98 & 0.97 & 0.99 & $<0.001$ & 1.12 & 1.08 & 1.15 & $<0.001$ \\
\hline 2 & 1.01 & 1.00 & 1.02 & 0.06 & 0.59 & 0.56 & 0.61 & $<0.001$ \\
\hline \multicolumn{9}{|l|}{ GCS in ED } \\
\hline 15 & [ref] & & & & [ref] & & & \\
\hline 14 & 1.21 & 1.20 & 1.23 & $<0.001$ & 0.62 & 0.60 & 0.64 & $<0.001$ \\
\hline 13 & 1.39 & 1.37 & 1.42 & $<0.001$ & 0.41 & 0.38 & 0.44 & $<0.001$ \\
\hline Penetrating injury & 1.19 & 1.14 & 1.25 & $<0.001$ & 0.84 & 0.76 & 0.92 & $<0.001$ \\
\hline \multicolumn{9}{|l|}{ Diagnoses } \\
\hline Skull fracture, no ICH & [ref] & & & & [ref] & & & \\
\hline Skull fracture, ICH & 2.85 & 2.79 & 2.92 & $<0.001$ & 0.65 & 0.61 & 0.68 & $<0.001$ \\
\hline $\mathrm{EDH}$ & 2.96 & 2.83 & 3.09 & $<0.001$ & 0.66 & 0.60 & 0.72 & $<0.001$ \\
\hline $\mathrm{SDH}$ & 2.58 & 2.52 & 2.64 & $<0.001$ & 0.91 & 0.87 & 0.95 & $<0.001$ \\
\hline $\mathrm{SAH}$ & 2.36 & 2.31 & 2.41 & $<0.001$ & 1.22 & 1.17 & 1.28 & $<0.001$ \\
\hline $\mathrm{SAH}$ and SDH & 2.97 & 2.89 & 3.05 & $<0.001$ & 0.71 & 0.66 & 0.75 & $<0.001$ \\
\hline Cerebral contusion & 2.17 & 2.11 & 2.23 & $<0.001$ & 1.06 & 1.01 & 1.12 & 0.02 \\
\hline Other TBI & 1.22 & 1.19 & 1.25 & $<0.001$ & 1.06 & 1.01 & 1.11 & 0.01 \\
\hline Multiple diagnoses & 3.10 & 3.04 & 3.17 & $<0.001$ & 0.56 & 0.53 & 0.60 & $<0.001$ \\
\hline \multicolumn{9}{|l|}{ Facility trauma level } \\
\hline I & [ref] & & & & [ref] & & & \\
\hline$\|$ & 1.08 & 1.05 & 1.11 & $<0.001$ & 1.05 & 0.96 & 1.16 & 0.29 \\
\hline III & 1.09 & 1.05 & 1.12 & $<0.001$ & 1.08 & 0.99 & 1.18 & 0.07 \\
\hline IVN & 1.02 & 0.96 & 1.08 & 0.47 & 1.02 & 0.87 & 1.19 & 0.80 \\
\hline \multicolumn{9}{|l|}{ Facility type } \\
\hline Community & [ref] & & & & [ref] & & & \\
\hline Non-teaching & 1.02 & 0.96 & 1.08 & 0.48 & 0.97 & 0.85 & 1.10 & 0.63 \\
\hline
\end{tabular}


Table 4 continued

\begin{tabular}{|c|c|c|c|c|c|c|c|c|}
\hline & \multicolumn{4}{|c|}{ Admission to the ICU from the ED } & \multicolumn{4}{|c|}{ Overtriage among those admitted to ICU } \\
\hline & $\mathbf{R R}$ & $95 \%$ & & $P$ & $\mathbf{R R}$ & $95 \%$ & & $P$ \\
\hline University & 1.00 & 0.95 & 1.05 & 0.91 & 1.07 & 0.98 & 1.17 & 0.14 \\
\hline \multicolumn{9}{|l|}{ Facility size } \\
\hline$\leq 200$ beds & 0.90 & 0.85 & 0.95 & $<0.001$ & 1.08 & 0.95 & 1.22 & 0.24 \\
\hline $201-400$ beds & [ref] & & & & [ref] & & & \\
\hline $401-600$ beds & 0.96 & 0.93 & 1.00 & 0.03 & 0.85 & 0.77 & 0.95 & 0.004 \\
\hline$\geq 601$ beds & 0.92 & 0.87 & 0.96 & $<0.001$ & 0.89 & 0.78 & 1.01 & 0.08 \\
\hline \multicolumn{9}{|l|}{ Facility ICU size } \\
\hline $1-10$ & [ref] & & & & [ref] & & & \\
\hline $11-25$ & 0.95 & 0.90 & 1.00 & 0.03 & 1.02 & 0.86 & 1.22 & 0.80 \\
\hline$>25$ & 1.02 & 0.97 & 1.07 & 0.47 & 1.04 & 0.86 & 1.26 & 0.66 \\
\hline \multicolumn{9}{|l|}{ Facility region } \\
\hline Northeast & [ref] & & & & [ref] & & & \\
\hline Midwest & 1.16 & 1.02 & 1.33 & 0.02 & 0.88 & 0.78 & 0.99 & 0.04 \\
\hline West & 1.22 & 1.06 & 1.41 & 0.006 & 0.97 & 0.84 & 1.10 & 0.61 \\
\hline South & 1.28 & 1.12 & 1.46 & $<0.001$ & 0.80 & 0.70 & 0.91 & 0.001 \\
\hline
\end{tabular}

AIS abbreviated injury scale, $\mathrm{Cl}$ confidence interval, ED emergency department, EDH epidural hematoma, GCS Glasgow coma scale, ICH intracranial hemorrhage, ICU intensive care unit, $I P H$ intraparenchymal hemorrhage, $R R$ risk ration, $S A H$ subarachnoid hemorrhage, $S D H$ subdural hematoma, $T B I$ traumatic brain injury

overtriage definition) rather than a true requirement for intensive monitoring in an ICU setting. It is not clear whether the greater level of care required following discharge is the result of worse neurological outcomes among the elderly [23], prehospitalization difficulties in the home environment that may have led to TBI (e.g., poor balance leading to a fall in an elderly patient who is unsafe to return home), or some combination of both. Favoring the former explanation, it has been shown that study populations with older patients tend to have higher rates of mortality and neurosurgical intervention [8]. Greater medical complexity requiring protracted hospitalization in this population may also contribute, as older age and medical comorbidities have found to be associated with a need for ICU care among elective neurosurgical patients [24].

We found that a number of factors known to be associated with the risk of poor outcomes and the need for surgical intervention were associated with a greater likelihood of ICU admission and a lower likelihood of overtriage. For example, patients with abnormal GCS were significantly more likely to be triaged to the ICU, with a roughly $20 \%$ increase in risk for each point below 15 . They were also substantially less likely to be overtriaged. These findings align with the increasing incidence of surgical mass lesions and poor outcomes with declining GCS, even among patients with a GCS of 13 or greater [2, 8]. Similarly, the presence of hypotension or coagulopathy (iatrogenic or otherwise) was associated with greater likelihood of ICU admission and a lower likelihood of being overtriaged; both of these clinical findings have been associated with poor neurological outcomes, and anticoagulation has independently been linked with expansion of intracranial hematomas and the need for surgical evacuation $[8,25-28]$. These results are intuitive and suggest that some factors placing patients at greater risk of deterioration prompt triage to the ICU for close monitoring.

However, we did find that some patients with a low risk of requiring ICU-level care are also more likely to be admitted to the ICU. For example, patients with isolated SAH were more likely to meet our criteria for overtriage compared with patients with other types of intracranial hemorrhage. This finding is congruent with previous work, which has shown that patients with an isolated traumatic SAH are at very low risk of requiring intervention $[8-10,29,30]$.

We observed considerable variation between facilities with regard to the risk of ICU admission and overtriage. The median risk ratio for ICU admission was 1.67 , which indicates that taking a patient from a less aggressive to a more aggressive hospital would result in a $67 \%$ increase in the probability of ICU admission at least half of the time. For the purposes of comparison, this increase is greater than that observed for patients with GCS 13 compared with patients with a normal GCS. However, there were at most weak associations between these outcomes and facility characteristics, suggesting that the variability occurs mostly at the level of the individual facility, not between hospitals with different 

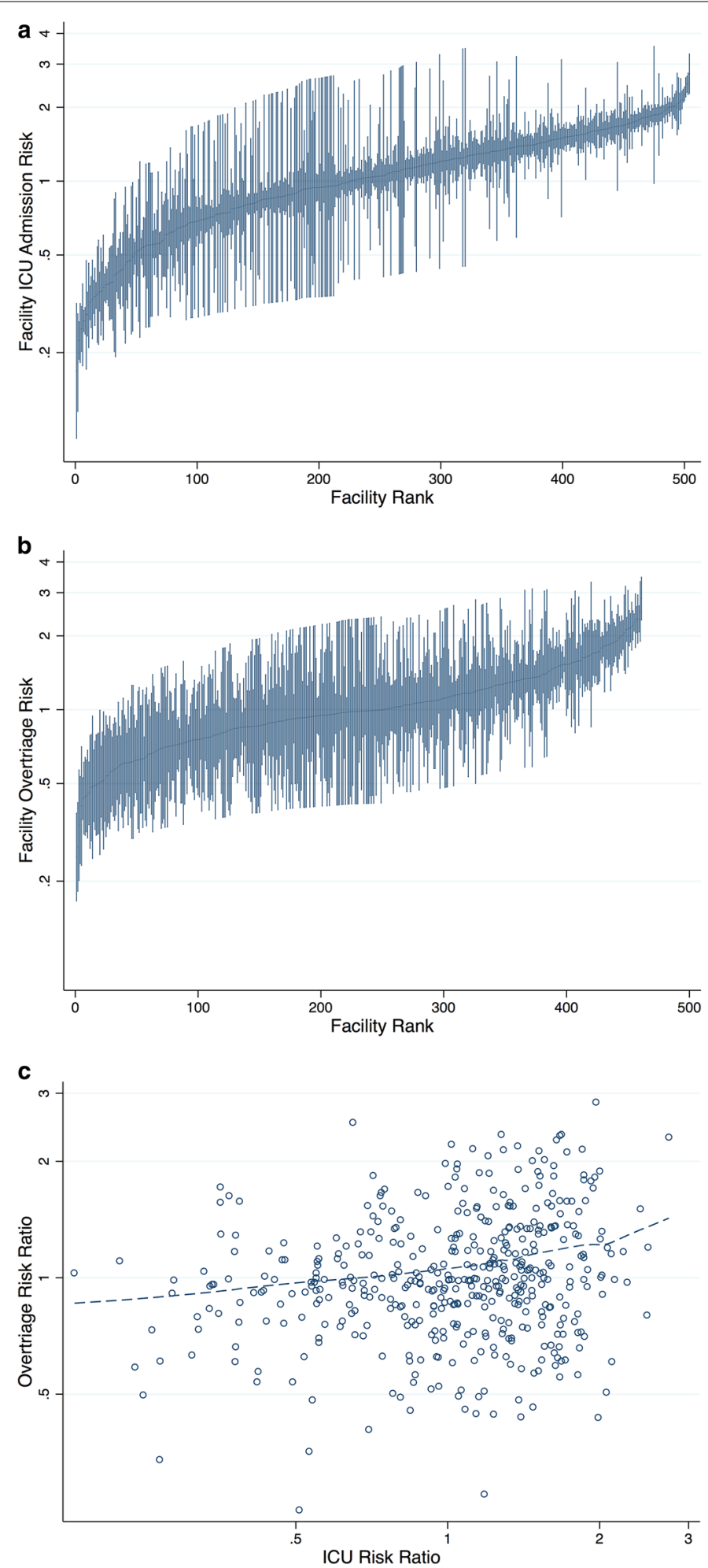

Fig. 1 Risk of ICU admission and overtriage at individual facilities. Panels A and B show the risk ratios with 95\% confidence intervals for ICU admission and overtriage, compared with the mean facility. Panel C shows a scatterplot comparing the risk of ICU admission to the risk of overtriage at each facility, with a dashed line representing the locally weighted scatterplot smoothing function. ICU intensive care unit 
trauma level designations, for example. It is possible that this inter-facility variation reflects differences in the acuity of patients cared for by different hospitals rather than discrepancies in local practice, but this seems unlikely. If this were true, patients receiving care at level 1 trauma centers should have the highest probability of being admitted to the ICU, but our data demonstrate that this is not the case.

Such facility-to-facility heterogeneity may be an ideal target to optimize resource utilization in the care of patients with mild TBI without compromising patient care. Indeed, ICU admissions require substantially more resources than observation in the ED or on the acute care ward, and as a consequence, they are considerably more expensive [31, 32]. Furthermore, ICU beds are limited, and overtriaging patients who do not require ICU-level observation to these units may lead to ICU over-crowding and the boarding of patients requiring intensive care elsewhere in the hospital [7]. Given the resource-intensive nature of ICU admissions, the rarity of clinical deterioration, and the lack of an outcome benefit in previous studies [4, 6-8], routine ICU admissions for all patients with mTBI may be unwarranted. Clinical decision rules have been developed in an attempt to identify mild TBI patients at low risk of requiring critical care or neurosurgical interventions [6]. Successful validation of such instruments could help to reduce the rates of unwarranted ICU admission without jeopardizing outcomes.

This study's strength lies in the use of a national dataset spanning several hundred hospitals. As such, we have been able to examine the patterns of ICU admission across facilities of varying characteristics. Furthermore, the large number of patients identified provides robust statistical power.

However, this study does have several limitations. While the NTDB does include GCS and intracranial injuries in the form of ICD-9-CM diagnostic codes, further specificity is lacking. For example, there is no information regarding trends in GCS over time, intracranial hematoma size, midline shift, or cisternal effacement, important indicators of brain injury that may be used by practitioners triaging these patients [5, 6, 33-35]. Furthermore, the diagnoses that are submitted to the NDTB by contributing hospitals are abstracted from the medical record at the conclusion of the patient's hospital stay; thus, injuries that were known at the time of discharge and included in our analysis may not have been apparent when triage decisions were made in the ED. Finally, ICD-9-CM diagnosis codes lack specificity with regard to intracranial injuries, and this weakness may be magnified by inconsistent coding practices across centers [36]. The extent to which this limitation influences our results is unclear.
The NDTB does not include patients who are not admitted to the hospital, which may also impact our findings. Some with mild TBI are discharged from the ED after a period of observation without an inpatient stay, a practice which-like ICU admission-may vary considerably from hospital to hospital [4]. Patients sent home from the ED would presumably have otherwise been admitted to acute care rather than the ICU, and their absence may influence comparisons between ICU- and floor-admitted patients, particularly at hospitals that frequently engage in this practice. The presence or absence of dedicated trauma and/or neuroscience ICUs at individual facilities may also influence triage patterns, but the NTDB does not provide information regarding the types of ICUs present at all hospitals.

Finally, our definition of "overtriage" to the ICU was constructed conservatively to identify patients whose TBI was sufficiently mild to warrant rapid discharge from the hospital without intubation or intervention. This strict definition likely omits a number of patients who were admitted to the ICU and did not require a critical care intervention. Indeed, only $17 \%$ of ICU-admitted patients in our study met the criteria for overtriage. By contrast, a recent meta-analysis found that the pooled prevalence of clinical deterioration was only $11 \%$, suggesting that $89 \%$ of patients would not have benefitted from close monitoring. Our approach allowed us to define an overtriaged group with the lowest likelihood of brain injuries requiring ICU-level care. It is possible that some patients in this group did require a critical care intervention or benefit from close monitoring during their brief ICU stays, but we are limited in our ability to examine this because the NTDB does not contain detailed information regarding all such interventions and observations. By including length of stay in our definition of overtriage, we may also have biased our comparison between the probabilities of ICU admission and overtriage toward the null; hospitals that more aggressively admit patients to the ICU may also be more conservative and cautious when it comes to discharge, leading to longer hospital stays and a lower probability of overtriage.

\section{Conclusion}

Using a large national dataset, we identified a number of patient characteristics that are associated with an increased probability of ICU admission, some of which were also associated with ICU admissions that may not have been necessary. Our data also suggest that there is considerable variability between facilities with regard to ICU admission practices, though hospitals that more aggressively triaged patients to the ICU did not necessarily have more unnecessary ICU admissions. A subset of mTBI patients may benefit from close observation in the 
ICU, but a substantial portion can probably be observed safely in an acute care setting, or possibly even at home. Further work will be required to help clinicians make safe decisions regarding triage from the ED.

\author{
Author details \\ ${ }^{1}$ Harborview Injury Prevention Research Center, Harborview Medical Center, \\ Seattle, WA, USA. ${ }^{2}$ Department of Neurological Surgery, University of Washing- \\ ton, Seattle, WA, USA. ${ }^{3}$ Department of Environmental \& Occupational Health, \\ Drexel University, Philadelphia, PA, USA. ${ }^{4}$ Department of Pediatrics, University \\ of Washington, Seattle, WA, USA. ${ }^{5}$ Department of Anesthesiology \& Pain \\ Medicine, University of Washington, Seattle, WA, USA.
}

\section{Author Contributions}

RHB drafted the manuscript, designed the study, and conducted the analysis. AQ contributed to the manuscript, designed the study, and conducted the analysis. FPR and MSV contributed to drafting of the manuscript and to study design.

\section{Source of Support}

RHB received partial, indirect salary support via an educational grant provided to the University of Washington Department of Neurological Surgery by Codman Neuro, who had no role in the design, conduct, or reporting of this research.

\section{Compliance with Ethical Standards}

Conflict of interest

The authors report no conflicts of interest.

Published online: 22 August 2018

\section{References}

1. Faul M, Xu L, Wald MM, Coronado V. Traumatic brain injury in the United States: emergency department visits, hospitalizations and deaths 2002-2006. Atlanta: Centers for Disease Control and Prevention, National Center for Injury Prevention and Control; 2010.

2. Teasdale G, Maas A, Lecky F, Manley G, Stocchetti N, Murray G. The Glasgow Coma Scale at 40 years: standing the test of time. Lancet Neurol. 2014;13(8):844-54.

3. Yun BJ, Borczuk P, Wang L, Dorner S, White BA, Raja AS. Evaluation of a low-risk mild traumatic brain injury and intracranial hemorrhage emergency department observation protocol. Acad Emerg Med. 2017;61(43):113.

4. Ratcliff JJ, Adeoye O, Lindsell CJ, et al. ED disposition of the Glasgow Coma Scale 13 to 15 traumatic brain injury patient: analysis of the transforming research and clinical knowledge in TBI study. Am J Emerg Med. 2014;32(8):844-50.

5. Ament JD, Greenan KN, Tertulien P, Galante JM, Nishijima DK, Zwienenberg M. Medical necessity of routine admission of children with mild traumatic brain injury to the intensive care unit. J Neurosurg Pediatr. 2017:19(6):668-74.

6. Nishijima DK, Sena M, Galante JM, et al. Derivation of a clinical decision instrument to identify adult patients with mild traumatic intracranial hemorrhage at low risk for requiring ICU admission. Ann Emerg Med. 2014;63(4):448-56.e2.

7. Nishijima DK, Haukoos JS, Newgard CD, et al. Variability of ICU use in adult patients with minor traumatic intracranial hemorrhage. Ann Emerg Med. 2013;61(5):509-517.e4.

8. Marincowitz C, Lecky FE, Townend W, Borakati A, Fabbri A, Sheldon TA The risk of deterioration in GCS13-15 patients with traumatic brain injury identified by computed tomography imaging: a systematic review and meta-analysis. J Neurotrauma. 2018;35(5):703-18.
9. Phelan HA, Richter AA, Scott WW, et al. Does isolated traumatic subarachnoid hemorrhage merit a lower intensity level of observation than other traumatic brain injury? J Neurotrauma. 2014;31(20):1733-6.

10. Borczuk P, Penn J, Peak D, Chang Y. Patients with traumatic subarachnoid hemorrhage are at low risk for deterioration or neurosurgical intervention. J Trauma Acute Care Surg. 2013;74(6):1504-9.

11. Pruitt P, Penn J, Peak D, Borczuk P. Identifying patients with mild traumatic intracranial hemorrhage at low risk of decompensation who are safe for ED observation. Am J Emerg Med. 2017:35(2):255-9.

12. Greene NH, Kernic MA, Vavilala MS, Rivara FP. Validation of ICDPIC software injury severity scores using a large regional trauma registry. Inj Prev. 2015;21(5):325-30.

13. Marr AL, Coronado VG. Central nervous system injury surveillance data submission standards-2002. Atlanta: Centers for Disease Control and Prevention, National Center for Injury Prevention and Control; 2004.

14. Maas AIR, Stocchetti N, Bullock R. Moderate and severe traumatic brain injury in adults. Lancet Neurol. 2008;7(8):728-41.

15. Rimel RW, Giordani B, Barth JT, Boll TJ, Jane JA. Disability caused by minor head injury. Neurosurgery. 1981;9(3):221-8.

16. Jagoda AS, Bazarian JJ, Bruns JJ, et al. Clinical policy: neuroimaging and decisionmaking in adult mild traumatic brain injury in the acute setting. Ann Emerg Med. 2008:52(6):714-48.

17. Nates JL, Nunnally M, Kleinpell R, et al. ICU admission, discharge, and triage guidelines: a framework to enhance clinical operations, development of institutional policies, and further research. Crit Care Med. 2016;44(8):1553-602

18. Kleinman ME, Chameides L, Schexnayder SM, et al. Part 14: pediatric advanced life support: 2010 American heart association guidelines for cardiopulmonary resuscitation and emergency cardiovascular care. Circulation. 2010;122(18 Suppl 3):S876-908.

19. Larson DB, Johnson LW, Schnell BM, Goske MJ, Salisbury SR, Forman HP. Rising use of $C T$ in child visits to the Emergency Department in the United States, 1995-2008. Radiology. 2011:259(3):793-801.

20. Rabe-Kesketh S, Skrondal A. Multilevel and longitudinal modeling using Stata, volume 2: categorical responses, counts, and survival. College Station: Stata Press; 2012

21. Austin PC, Stryhn H, Leckie G, Merlo J. Measures of clustering and heterogeneity in multilevel Poisson regression analyses of rates/count data. Stat Med. 2018;37(4):572-89.

22. Myers SR, Branas CC, French B, Nance ML, Carr BG. A national analysis of pediatric trauma care utilization and outcomes in the United States. Pediatr Emerg Care 2016. https://doi.org/10.1097/PEC.000000000000090 2.

23. Marshall LF, Gautille T, Klauber MR, et al. The outcome of severe closed head injury. J Neurosurg. 1991;75:S28-36.

24. Hanak BW, Walcott BP, Nahed BV, et al. Postoperative intensive care unit requirements after elective craniotomy. World Neurosurg. 2014;81(1):165-72.

25. Chesnut RM, Marshall SB, Piek J, Blunt BA, Klauber MR, Marshall LF. Early and late systemic hypotension as a frequent and fundamental source of cerebral ischemia following severe brain injury in the Traumatic Coma Data Bank. Acta Neurochir Suppl. 1993;59:121-5.

26. Ivascu FA, Howells GA, Junn FS, Bair HA, Bendick PJ, Janczyk RJ. Rapid warfarin reversal in anticoagulated patients with traumatic intracranial hemorrhage reduces hemorrhage progression and mortality. J Trauma. 2005;59(5):1131-7.

27. Moore MM, Pasquale MD, Badellino M. Impact of age and anticoagulation: need for neurosurgical intervention in trauma patients with mild traumatic brain injury. J Trauma Acute Care Surg. 2012;73(1):126-30.

28. Grandhi R, Harrison $G$, Voronovich Z, et al. Preinjury warfarin, but not antiplatelet medications, increases mortality in elderly traumatic brain injury patients. J Trauma Acute Care Surg. 2015;78(3):614-21.

29. Levy AS, Orlando A, Hawkes AP, Salottolo K, Mains CW, Bar-Or D. Should the management of isolated traumatic subarachnoid hemorrhage differ from concussion in the setting of mild traumatic brain injury? J Trauma. 2011:71(5):1199-204.

30. Quigley MR, Chew BG, Swartz CE, Wilberger JE. The clinical significance of isolated traumatic subarachnoid hemorrhage. J Trauma Acute Care Surg. 2013;74(2):581-4. 
31. Milbrandt EB, Kersten A, Rahim MT, et al. Growth of intensive care unit resource use and its estimated cost in Medicare. Crit Care Med. 2008;36(9):2504-10.

32. Halpern NA, Pastores SM. Critical care medicine in the United States 2000-2005: an analysis of bed numbers, occupancy rates, payer mix, and costs. Crit Care Med. 2010;38(1):65-71.

33. Lingsma HF, Yue JK, Maas AIR, Steyerberg EW, Manley GT, TRACK-TBI Investigators. Outcome prediction after mild and complicated mild traumatic brain injury: external validation of existing models and identification of new predictors using the TRACK-TBI pilot study. J Neurotrauma. 2015:32(2):83-94
34. MRC CRASH Trial Collaborators, Perel P, Arango M, et al. Predicting outcome after traumatic brain injury: practical prognostic models based on large cohort of international patients. BMJ. 2008;336(7641):425-9.

35. Jacobs B, Beems T, Stulemeijer M, et al. Outcome prediction in mild traumatic brain injury: age and clinical variables are stronger predictors than CT abnormalities. J Neurotrauma. 2010;27(4):655-68.

36. Arabian SS, Marcus M, Captain K, et al. Variability in interhospital trauma data coding and scoring: a challenge to the accuracy of aggregated trauma registries. J Trauma Acute Care Surg. 2015;79(3):359-63. 